\title{
Systematic Investigations of the ZF-HD Gene Family in Tobacco Reveal Their Multiple Roles in Abiotic Stresses
}

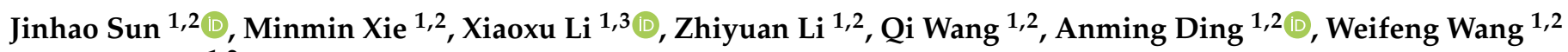 \\ and Yuhe Sun $1,2, *$ \\ 1 Key Laboratory for Tobacco Gene Resources, Tobacco Research Institute, Chinese Academy of Agricultural \\ Sciences, Qingdao 266101, China; $82101181072 @$ caas.cn (J.S.); xieminmin@caas.cn (M.X.); \\ 82101171073@caas.cn (X.L.); 82101181071@caas.cn (Z.L.); 82101181074@caas.cn (Q.W.); \\ dinganming@caas.cn (A.D.); wangweifeng@caas.cn (W.W.) \\ 2 Graduate School of Chinese Academy of Agricultural Science, Beijing 100081, China \\ 3 Technology Center, China Tobacco Hunan Industrial Co., Ltd., Changsha 410007, China \\ * Correspondence: sunyuhe@caas.cn; Tel./Fax: +86-532-8870-2056
}

Citation: Sun, J.; Xie, M.; Li, X.; Li, Z.; Wang, Q.; Ding, A.; Wang, W.; Sun, Y. Systematic Investigations of the ZF-HD Gene Family in Tobacco Reveal Their Multiple Roles in Abiotic Stresses. Agronomy 2021, 11, 406. https://doi.org/10.3390/ agronomy11030406

Academic Editor: Alejandro Galindo

Received: 24 December 2020

Accepted: 18 February 2021

Published: 24 February 2021

Publisher's Note: MDPI stays neutral with regard to jurisdictional claims in published maps and institutional affiliations.

Copyright: (c) 2021 by the authors. Licensee MDPI, Basel, Switzerland. This article is an open access article distributed under the terms and conditions of the Creative Commons Attribution (CC BY) license (https:/ / creativecommons.org/licenses/by/ $4.0 /)$.

\begin{abstract}
Zinc finger homeodomain (ZF-HD) transcription factors play significant roles in plant growth and responses to environmental stresses. In this study, $32 \mathrm{ZF-HD}$ genes identified in the tobacco (Nicotiana tabacum L.) genome were divided into six groups according to phylogenetic analysis with Arabidopsis and tomato ZF-HD members. An examination of gene structures and conserved motifs revealed the relatively conserved exon/intron structures and motif organization within each subgroup. In addition, various stress-related elements are found in the promoter region of these genes. The expression profiling analysis revealed that NtZF-HD genes expressed in different tissues and could be induced by several abiotic stresses. Notably, NtZF-HD21 was highly expressed in response to the drought treatments. Subcellular localization analysis and a virus-induced gene silencing (VIGS) experiment were performed to investigate the potential functions of NtZF-HD21. The subcellular localization indicated that NtZF-HD21 is a nuclear protein. Furthermore, gene silencing of the NtZF-HD21 gene reduced the drought resistance of tobacco. These findings provide insights for further biological functional analyses of the NtZF-HD genes in tobacco.
\end{abstract}

Keywords: Nicotiana tabacum; ZF-HD genes; genome-wide analysis; abiotic stresses

\section{Introduction}

Transcription factors (TFs), as a special class of proteins with the activity of binding to specific regions of target gene promoters, play significant roles in plant growth and development, stress resistance, and signal transduction [1-3]. Zinc finger homeodomain (ZF-HD) TFs usually include the following two structural features: a cysteine-rich zinc finger motif (ZF) and a conserved homeodomain (HD) [3]. ZFs contain zinc ions and cysteine residues or histidine residues [4]. ZFs are widely found in diverse regulatory proteins, which can specifically bind to DNA/RNA sequences and be actively involved in protein-protein interactions [5,6]. The HD, as a DNA-binding domain (BD), consists of about 60 amino acids that fold into a recognition helix that is able to specifically bind DNA to activate or suppress the expression of target genes [7-11]. The proteins with an HD are classified into six subgroups according to the presence of structural differences: leucine zipper-associated HD (HD-ZIP), zinc finger motif-associated HD (ZF-HD), WUSCHELrelated homeobox (WOX), Bell-type HD, finger domain associated to a HD (PHD finger) and Knotted-related homeobox (KNOX) proteins [12].

Previous research studies revealed that $Z F-H D$ genes participate in many essential biological processes in plants [13,14]. In Arabidopsis, a total of 17 ZF-HD genes were identified, and the contributions of a number of $Z F-H D$ s to various developmental processes have been characterized [15-17]. For instance, overexpressing transgenic plants of AtZF-HD5 
reportedly exhibit a larger size of leaves [18]. AtZF-HD10 was detected highly expressed in the hypocotyl axis in Arabidopsis and induced the expression of the hypocotyl-elongationrelated genes HFR1 (LONG HYPOCOTYL IN FAR-RED) and ATXTH17 (XYLOGLUCAN ENDOTRANSGLUCOSYLASE/HYDROLASE 17) [19]. AtZF-HD8, expressed highly in flower, revealed that they dominate significant roles in the development of flowering [7]. In other species, it was also found that ZF-HD genes have important roles in leaf development and flower bud development, such as SIZF-HD7 of tomato and FtZF-HD11 in Tartary buckwheat (Fagopyrum tataricum) [20,21].

Furthermore, a large number of ZF-HD genes were found to respond to abiotic stresses in plants [19,22-24]. AtZF-HD1 encoded a positive transcriptional regulator that was reported to specifically bind to the ERD1 (Early Response to Dehydration Stress 1) promoter region, whose expression was induced by salinity, dehydration, and ABA (abscisic acid) treatments [22]. AtZF-HD4 could be induced under drought and salt treatments, implying that AtZF-HD4 responds to abiotic stresses in Arabidopsis [25]. Previous studies indicated that AtZF-HD10 interacted with TANDEM ZINC-FINGER PLUS3 (TZP) to modulate hormone signaling in stress response [19]. More recently, a study showed that GmZFHD1 and GmZF-HD2 can bound to the promoter of GmCaM4 (Calmodulin Subtype 4) in soybean, and the expressions of GmZF-HD1 and GmZF-HD2 were up-regulated upon pathogen inoculation [26]. Additionally, the ZF-HD genes were involved in abiotic stress in tomato and Chinese cabbage [3,20].

Tobacco is a significant economic crop and a representative model plant widely cultivated all over the world. Biotic and abiotic stresses can severely decrease tobacco quality and yield. Although the ZF-HD gene family has been extensively studied in many species, such as Arabidopsis [11], tomato [20], rice [27], and apple [28], systematic and comprehensive identification had not been conducted in tobacco. Previous studies provided the necessary information for the data-predicted NtZF-HD gene family in tobacco due to the improved annotation of genome-wide sequencing of tobacco $[29,30]$. In this study, a comprehensive genome-wide investigation and expression analysis of the NtZF-HD gene family in tobacco were performed, including phylogenetic analyses, protein domain organization, gene structure, promoter analysis, expression pattern, and VIGS analysis. The results of this study indicate that the tobacco ZF-HD gene family members might play multiple roles in various biological processes, including development and responses to various stresses.

\section{Materials and Methods}

\subsection{Identification of Tobacco ZF-HD Proteins}

The genome data of the tobacco (N. tabacum), N. sylvestris, N. tomentosiformis and tomato (Solanum lycopersicum) were downloaded from the Sol Genomics Network (https: //solgenomics.net/) (accessed on 21 October 2019) [31] and the genome data of the Arabidopsis were downloaded from TAIR (http:/ / www.arabidopsis.org/) (Accessed 21 October 2019) [32]. The previously reported sequences of Arabidopsis and tomato ZF-HD full-length protein were used as queries to carry out BLASTP searches against the tobacco annotation database under the E-value cutoff of 0.01 . Then, the resulting sequences were subjected to the Pfam (PF04770) [33] and SMART (http:/ / smart.embl-heidelberg.de/) (accessed on 21 October 2019) [34] databases to determine the number of the ZH-FD domain. After removing the incomplete sequences and repeating manually, the remaining numbers were named based on their physical locations on the chromosome/scaffold and were submitted to the ProtParam online toolkits (http:/ / au.expasy.org/tools/protparam.html) (accessed on 21 October 2019) for the calculation of Biochemical characteristics, including Mw (molecular weight), theoretical pI (isoelectricpoint), and subcellular localization [35].

\subsection{Phylogenetic Analysis and Classification}

The proteins of tobacco, N. sylvestris, N. tomentosiformis, Arabidopsis, and tomato ZF-HD members, as shown in the Table S1, were aligned using ClustalX 2.0. The phylogenetic tree 
was constructed using the Neighbor-Joining (NJ) method in MEGA 7 with the p-distance method and pairwise deletion setting. A bootstrap statistical analysis was conducted with 1000 replicates to detect the reliability of each branch [36].

\subsection{Gene Structure, Motif Analysis, and Cis-Elements Analyses}

The gene structure of tobacco ZF-HD genes was analyzed by GSDS (http://gsds. cbi.pku.edu.cn) (accessed on 21 October 2019) using the coding sequence (CDS) [37] and genomic sequence. The online site Multiple Em for Motif Elicitation (MEME; http:/ / memesuite.org/) (accessed on 21 October 2019) was applied to identify conserved motifs of the ZF-HD full-length protein sequences [38]. The promoter region of tobacco ZF-HD genes was extracted by Promoter 2.0, and the cis-elements of promoters were analyzed by PlantCARE (http:/ / bioinformatics.psb.ugent.be/webtools/plantcare/html/) (accessed on 21 October 2019) [39].

\subsection{Syntenic Analysis of NtZF-HD Genes}

To explore the syntenic relationship of the orthologous genes obtained from tobacco and other tested plant species (Arabidopsis, tomato, grape, rice, and maize), syntenic analysis maps were investigated by the Systeny Plotter of Tbtools (https:/ / github.com/CJChen/ TBtools) (accessed on 21 October 2019) [40].

\subsection{Plant Material and Stress Treatment}

Cultivated tobacco (Nicotiana tabacum L. Cv. K326) plants were used in this study. Different organs, including the root, stem, leaf, and flower were used to analyze the tissuespecific expression patterns. The tobacco seeds were germinated on MS medium in a light incubator at $25^{\circ} \mathrm{C}$ for two weeks. The tobacco seedlings were transferred and exposed to drought and salt stress treatment for 0,3 , and $6 \mathrm{~h}$. These treatments were performed as previously described by Khatun et al. with some modification [41]. Drought stress treatment involved transferring seedlings to dry paper at 0,3 , and $6 \mathrm{~h}$. Roots of seedlings were submerged in $150 \mathrm{mM} \mathrm{NaCl}$ during salt stress treatment at 0,3 , and $6 \mathrm{~h}$. After different treatments, leaf samples from three biological replicates were collected and frozen in liquid nitrogen immediately and transferred to $-80{ }^{\circ} \mathrm{C}$ for RNA extraction.

\subsection{RNA Extraction and RT-qPCR Analysis}

Total RNA was extracted using the method of Ren et al. [42] and the first-strand complementary DNA (cDNA) was synthesized using the PrimeScript ${ }^{\mathrm{TM}}$ RT reagent Kit (TaKaRa). Using Primer3 software (http:/ / bioinfo.ut.ee/primer3/) (accessed on 21 October 2019) [43], we designed the RT-qPCR primers (Table S2). RT-qPCR was performed on an ABI7500 Real-Time PCR System (Applied Biosystems, Foster City, CA, USA) with $2 \mu \mathrm{L}$ template cDNA. The internal control used was the transcript of tobacco ribosomal protein gene L25 (GenBank No. L18908). At least three biological replications and three technical repeats of all reactions were performed, and the relative expression levels of each gene was analyzed using the $2^{-\Delta \Delta C T}$ method [44].

\subsection{Subcellular Localization}

The subcellular localization of NtZF-HD proteins was predicted using the online PlantmPLoc server (http:/ / www.csbio.sjtu.edu.cn/bioinf/plant-multi/) (accessed on 21 October 2019) [45]. The coding regions of NtZF-HD21 without the stop codon were amplified from tobacco cDNA and inserted into the PYG57 vector, generating the 35S::NtZF-HD21GFP fusion construct. According to the previous study [46], the recombinant 35S::NtZFHD21-GFP vector and the control vector were transformed into Agrobacterium competent cell GV3101 and then injected in the leaves of N. benthamiana. After the incubation, the confocal microscope (TCS-SP8 Leica, Wetzlar, Germany) was used to investigate Green Fluorescent Protein (GFP) signal under a $488 \mathrm{~nm}$ exciting light, and the nuclear localization signal was confirmed by 4,6-diamidino-2-phenylindole (DAPI) dye staining. 


\subsection{VIGS (Virus-Induced Gene Silencing)}

The VIGS vector system including TRV2 (empty vector), TRV1 (auxiliary vector), and $T R V 2:: P D S$ (positive control) was used in this experiment. The conserved sequences of NtZF-HD21 gene were selected from the SGN-VIGS website (https:/ / vigs.solgenomics. net/) (accessed on 21 October 2019) [47]. The conserved sequences were inserted into the TRV2 vector with the KpnI site and EcoRI site. The new vector named as TRV2::NtZF-HD21 was introduced into an Agrobacterium competent cell GV3101. The detailed VIGS operating methods were performed as previously described by Gao et al. (2013) [48].

\section{Results}

\subsection{Identification of NtZHD Genes in Tobacco}

To identify NtZF-HD family genes, we used the ZF-HD numbers of Arabidopsis as queries to the BLASTP search. A total of $32 \mathrm{ZF-HD}$ genes were identified in tobacco and designated NtZF-HD1 to NtZF-HD32 based on their physical locations on the chromosomes (Table S3). The results showed that $12 \mathrm{NtZF-HDs}$ were mapped on seven chromosomes and $20 \mathrm{NtZF-HDs}$ were localized on scaffolds.

As shown in Table S3, the ORF (open reading frame) lengths of the NtZF-HD genes range from $417 \mathrm{bp}$ (NtZF-HD28) to $3078 \mathrm{bp}$ (NtZF-HD2). Their isoelectric points (pIs) range from 6.37 (NtZF-HD25) to 10.25 (NtZF-HD28), and their molecular weights (MWs) range from 15.59 (NtZF-HD28) to $113.63 \mathrm{kDa}$ (NtZF-HD2).

\subsection{Phylogenetic Analysis of the NtZF-HD Family Members}

To further elucidate the phylogenetic relationships of the NtZF-HD family proteins, a neighbor-joining (NJ) tree was constructed based on the multiple sequence alignment of the 32 tobacco ZF-HD members and their homologs in Arabidopsis thaliana, N. sylvestris, $N$. tomentosiformis, rice, and tomato (Figure 1). The 32 NtZF-HD members were classified into eight groups (I-VIII), together with their Arabidopsis and tomato homologs based on previously reported [20,23]. Groups V and II contained the smallest (one) and largest (nine) number of proteins, respectively. Groups IV and VI both account for $19 \%$ of the total NtZF-HD proteins. Groups I and III both included three members of the NtZF-HD proteins. Only groups II, III, and IV contained rice members, suggesting that the member of these groups in the ZF-HD gene family occurred before the divergence of dicotyledon and monocotyledon. Notably, groups VII and VIII only contained tobacco and tomato members, suggesting that these ZF-HD numbers might occur after the divergence of Arabidopsis and were unique to Solanaceae plants. Interestingly, a large number of NtZF-HD members from different groups could be clustered with NtomZF-HD and NsylZF-HD members, implying that these species had a closer evolutionary relationship. However, in group V, NtZF-HD1 was clustered only with NsylZF-HD13 and NsylZF-HD17, suggesting that the NtomZF-HD from $N$. tomentosiformis may be lost after interspecific hybridization.

\subsection{Structural Analysis and Motif Composition of the NtZF-HD Family}

The multigene family analysis of genetic structural diversity can provide important insights into the evolutionary information of NtZF-HD genes. To further explore the evolution of the ZF-HD genes in tobacco, the number and arrangement of their exon-intron structures were identified (Figure S2). The members of different groups exhibited different exon-intron organization. Among the $32 \mathrm{NtZF-HD} \mathrm{genes,} \mathrm{there} \mathrm{were} 15 \mathrm{NtZF-HD} \mathrm{genes}$ with intronless and clustered into groups III, IV, and V. The remaining 17 NtZF-HD genes coding sequences were interrupted by introns, and the number of exons varied from one to eight. The remaining NtZF-HD genes belonged to groups I, II, and VI (Figure S2B). The introns of the NtZF-HD gene were obtained by evolutionary inheritance or by mutations in the evolutionary process, and whether the emergence of introns affects the function of these genes is a worthy question for further research. 


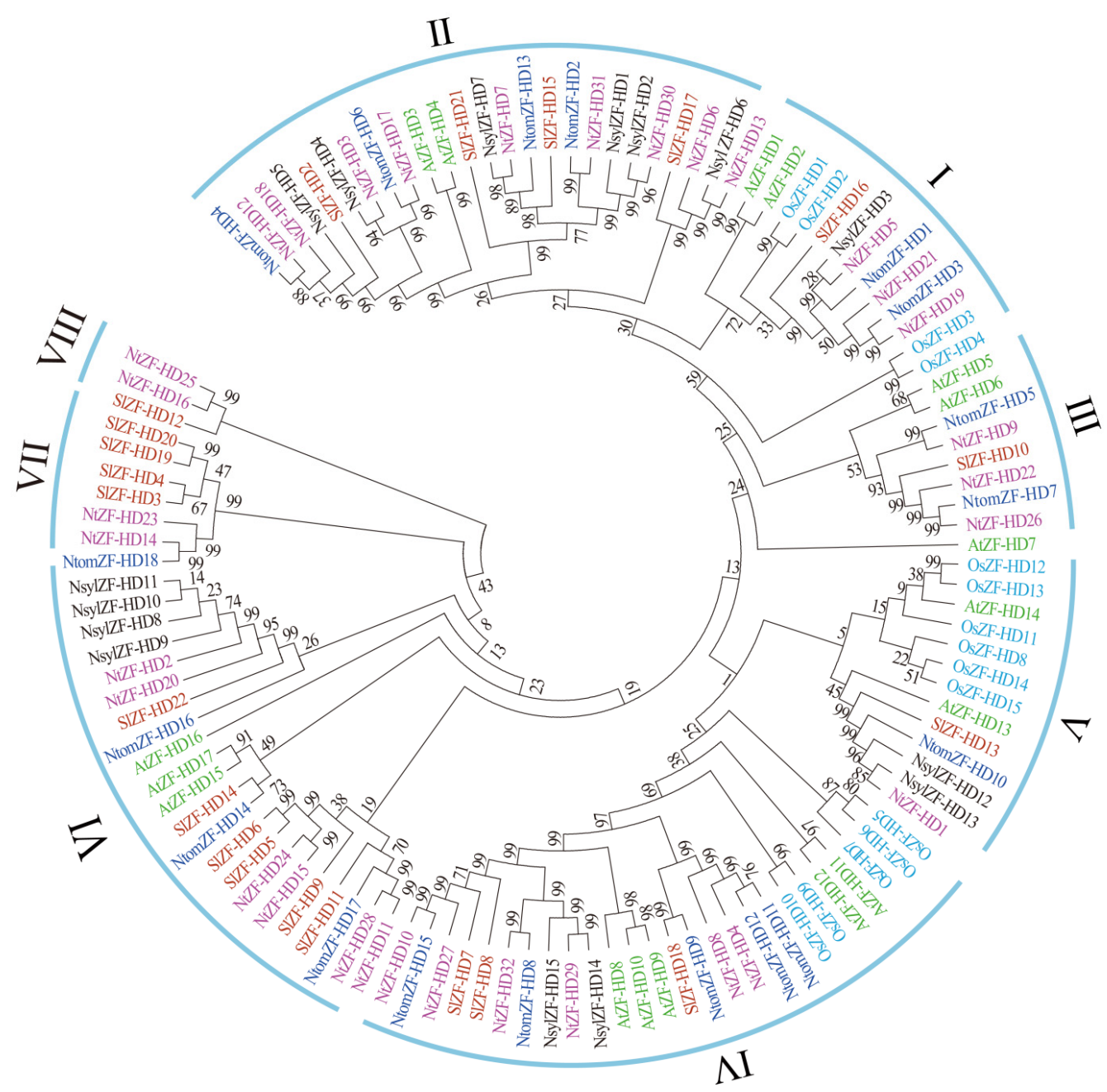

Figure 1. Phylogenetic relationship of Arabidopsis (AtZF-HD), tomato (SlZF-HD), rice (OsZF-HD), N. sylvestris (NsylZF-HD), N. tomentosiformis (NtomZF-HD), and tobacco (NtZF-HD) ZF-HD members, divided into six groups. The phylogenetic tree was generated using MEGA7 with the neighbor-joining method. The number of bootstrap values was set to 1000 replications. ZF-HD: Zinc finger homeodomain.

To further investigate the structural characteristics of the ZF-HD proteins in tobacco of the ZF-HD proteins in tobacco, the conserved motifs of the 32 NtZF-HD, 17 AtZF-HD, and 22 SlZF-HD proteins were analyzed using the online MEME tool. A total of 10 conserved motifs were identified and named as motifs 1 to 10 (Figure S2C). These motifs contained 20 to 49 amino acids, and their amino acid sequences are shown in the Supplementary Table S4. The NtZF-HD proteins were clustered into the same group that usually has a similar motif distribution, which is consistent with that of the SIZF-HD proteins. Notably, all NtZF-HD proteins have motif 3, and all proteins except for NtZF-HD12 have motif 1 . Interestingly, similar conditions exist in SIZF-HD proteins, with only one protein, SIZF-HD1, without motif 1 . Moreover, some conserved motifs existed only in specific groups. For example, motif 5 and motif 6 were only presented in group IV with a high specificity, while they could only be found in groups II and III. The specific distribution of conserved motifs in each group may reflect the specific functions of NtZF-HD genes in tobacco.

\subsection{Syntenic Analysis of NtZF-HD Genes}

To further understand the evolutionary relationship among the tobacco ZF-HD genes, the syntenic analysis was carried out for tobacco and five other plant species, including dicotyledonous plants (Arabidopsis, tomato, and grape) and monocotyledonous plants (rice and maize) (Figure 2A). The results showed that there was a syntenic relationship between 
six of the NtZF-HD genes with ZF-HD genes in Arabidopsis, six ZF-HD genes in grape, four $Z F-H D$ genes in tomato, two ZF-HD genes in maize, and two ZF-HD genes in rice. The numbers of predicted collinear pairs between tobacco and Arabidopsis, tomato, grape, rice, and maize were seven, seven, six, three, and two, respectively. Meanwhile, only one ZF-HD gene (NtZF-HD5) was predicted to form collinear pairs with ZF-HD genes of all the other five species, suggesting that this ZF-HD gene may exist before the differentiation of these species and has maintained a collinear relationship since then. Interestingly, one collinear gene pair was identified between tobacco and tomato/grape/Arabidopsis species, but no collinear gene pairs were found in the rice and maize genome, indicating that this pair may have appeared after the divergence of dicot and monocot (Figure 2B, Table S5).

A
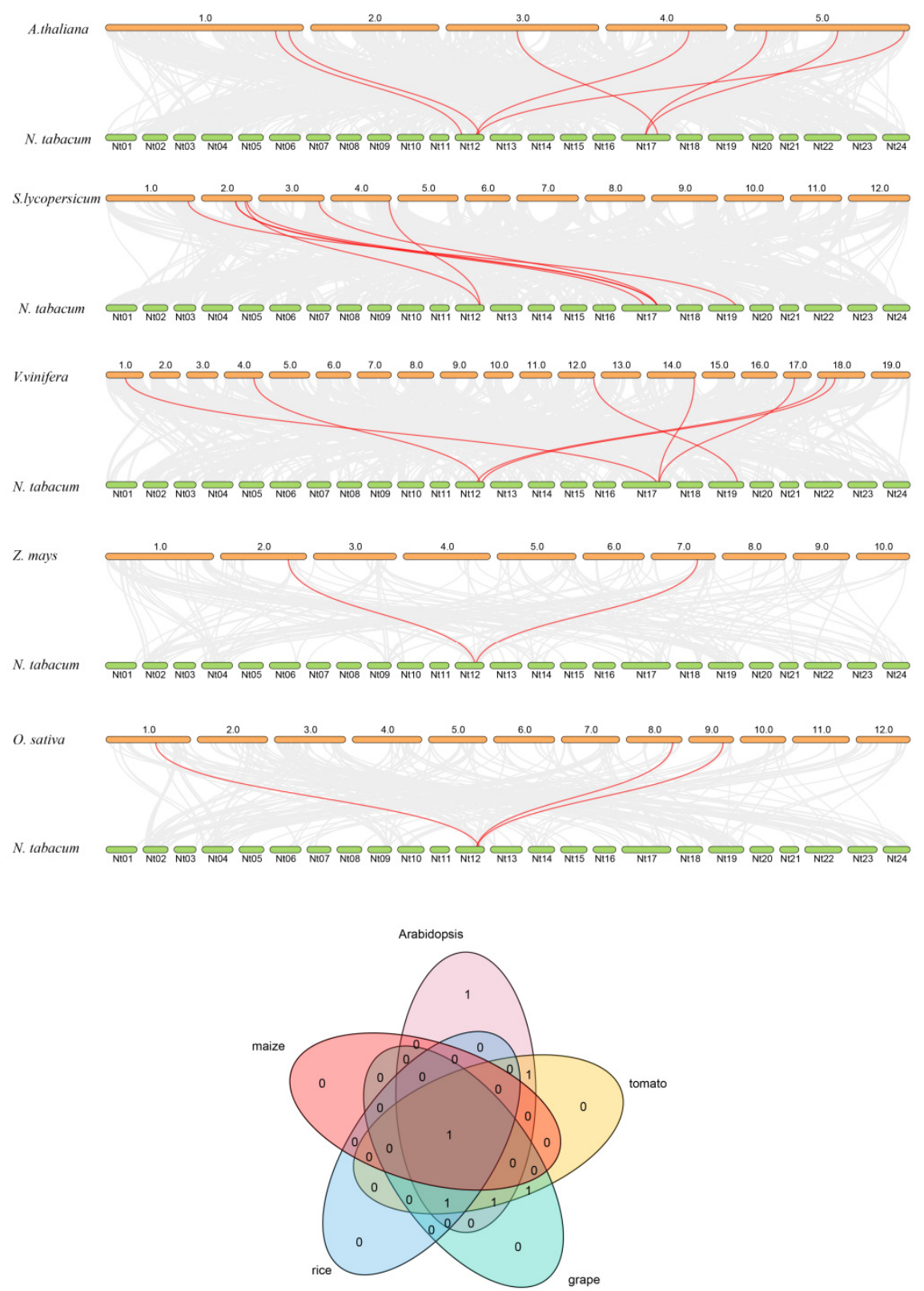

Figure 2. Syntenic analysis of ZF-HD genes between tobacco and five other representative species. (A) The gray line in the background represents the colinear blocks between tobacco and five other representative species, while the red line represents the syntenic ZF-HD gene pairs. (B) The numbers of ZF-HD genes that formed syntenic pairs between ZF-HD and the other four selected species, which was visualized by the Venn diagram. 


\subsection{Promoter Analysis of NtZF-HD Family Genes}

To further investigate the potential response patterns of ZF-HD genes, cis-elements in promoter regions of $32 \mathrm{NtZF-HD}$ gene were analyzed by the online PlantCARE tool (Figure 3, Table S6). One or more MYB binding sites (v-myb avian myeloblastosis viral oncogene homolog) were identified in promoters of all the NtZF-HD genes, except NtZFHD5 and NtZF-HD11. In addition, the hormone-response elements were also identified in promoter regions, including estrogen response element (ERE), abscisic acid response element (ABRE), TCA-element and CGTCA-motif. Furthermore, 17 of the NtZF-HD genes contain at least one wound-responsive element (WUN-motif), 15 of the NtZF-HD genes contain at least one anaerobic induction element (ARE), and 11 of the NtZF-HD genes contain at least one stress-responsive element (TC-rich repeats), implying that these NtZF$H D$ genes may be involved in various stress responses.

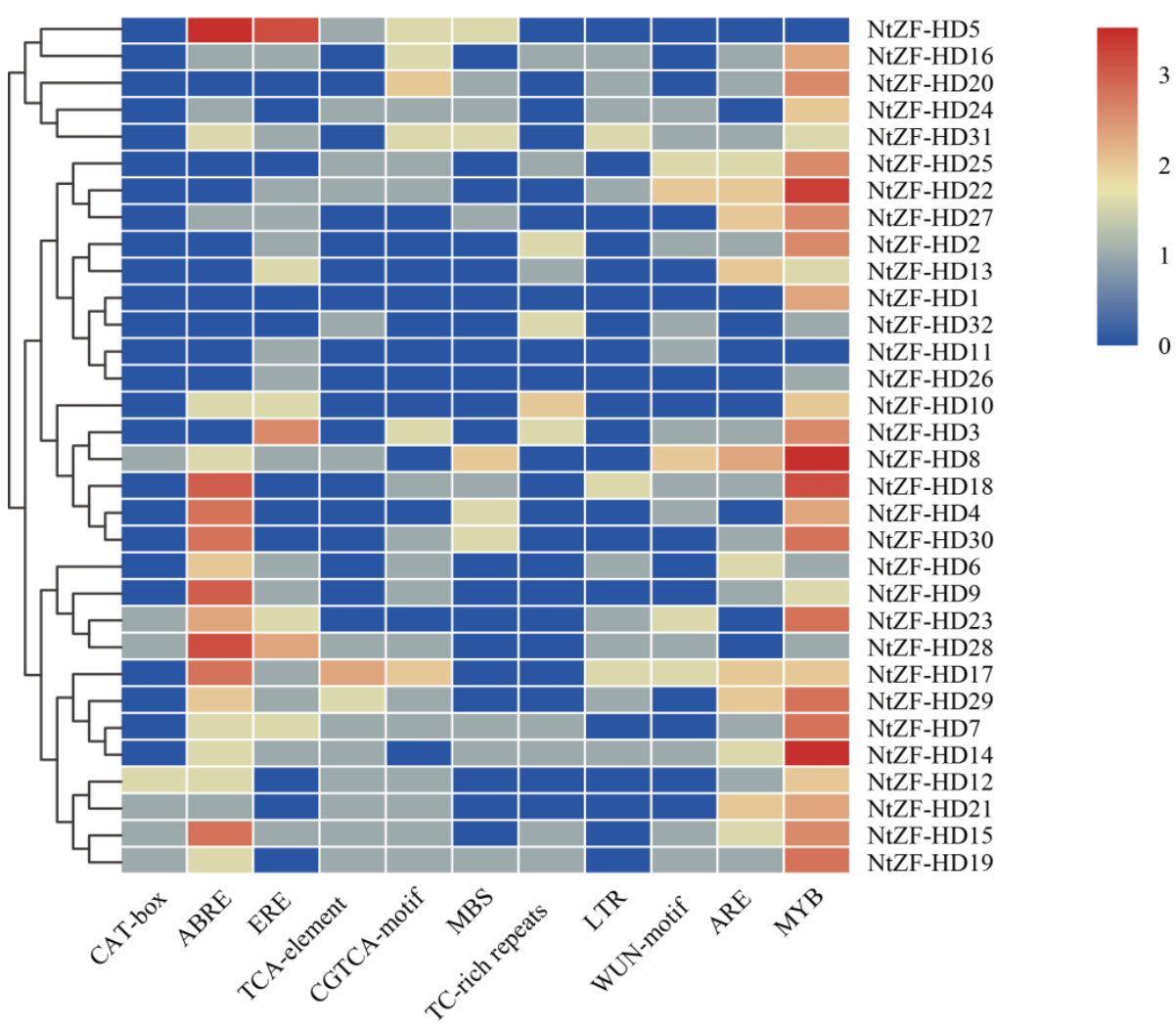

Figure 3. Statistics diagram of cis-elements in the promoter region of NtZF-HD genes. The colors represent the numbers of every cis-element in NtZF-HD family genes.

\subsection{Expression Profiles of NtZF-HD Family Genes}

To preliminarily elucidate the roles of NtZF-HD genes in tobacco growth and development, the relative expression levels of NtZF-HD genes in six different tissues including young leaves, mature leaves, aging leaves, roots, stems, and flowers were analyzed using RT-qPCR (Figure S2). A number of the NtZF-HD genes were expressed in a tissue-specific manner; for example, in group I, NtZF-HD19 was highly expressed in young leaves and flowers. In group II, NtZF-HD7 was only expressed in root, and the expression of NtZFHD30 was detected in the stems exclusively. NtZF-HD3, NtZF-HD13, and NtZF-HD16 were mainly expressed in all leaf stages. Similarly, NtZF-HD22 of group III was found to be highly expressed in aging leaves. Other tissue-specific expression patterns were also detected; in group VI, NtZF-HD11 and NtZF-HD25 were highly expressed in young leaves and flowers. The tissue-specific expression pattern of these genes may be involved in the mediation of various developmental processes of tobacco. 
In addition, to further investigate the expression patterns of tobacco NtZF-HD genes under abiotic stress, $16 \mathrm{NtZF-HD}$ genes were detected to determine their expression levels under drought and $\mathrm{NaCl}$ treatment (Figure 4A,B). Genes in group I (NtZF-HD21) and IV (NtZF-HD4 and NtZF-HD27) could be induced and up-regulated significantly under drought treatment. Furthermore, NtZF-HD12 and NtZF-HD18 in group II had up-regulations of the transcription levels under salt and drought treatment. Moreover, in group VIII, NtZF-HD16 and NtZF-HD25 were up-regulated under salt and drought treatments, whereas NtZF-HD24 in group VI was down-regulated by salt and drought treatments. These expression analyses would provide a valuable resource for researching the roles of NtZF-HD genes in control of the stress responses.
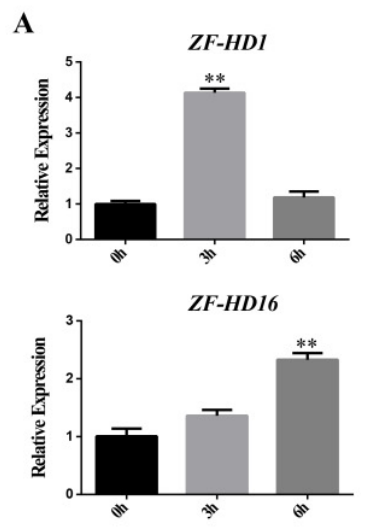

ZF-HD24

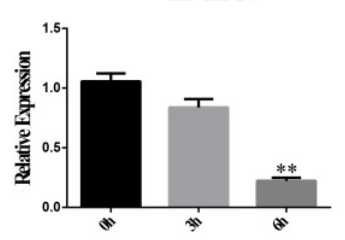

B

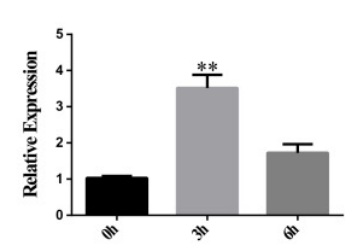

ZF-HD16

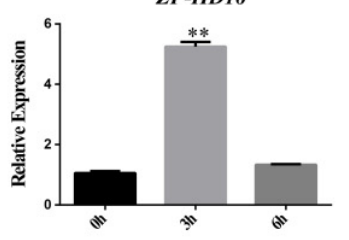

ZF-HD24

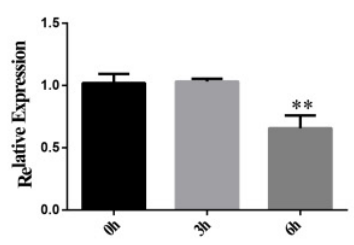

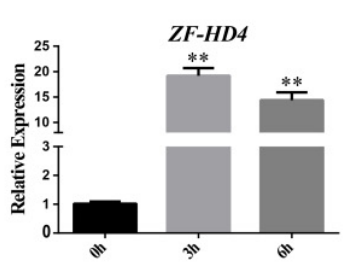

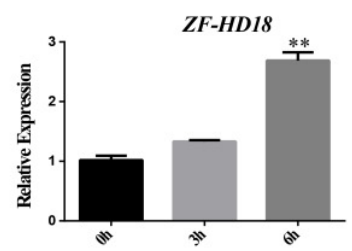

ZF-HD25

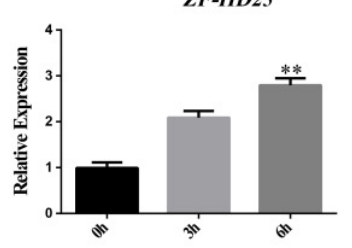

ZF-HD4

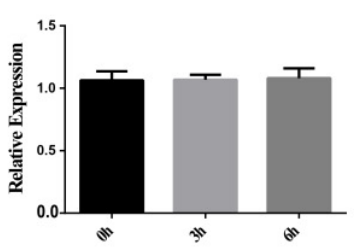

ZF-HD18

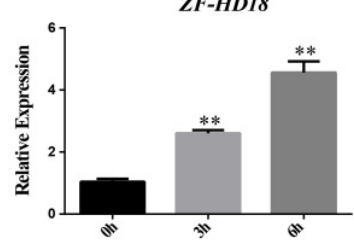

ZF-HD25

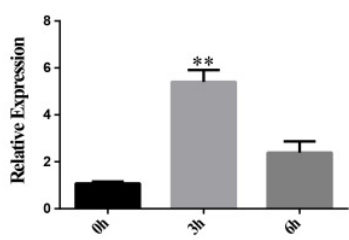

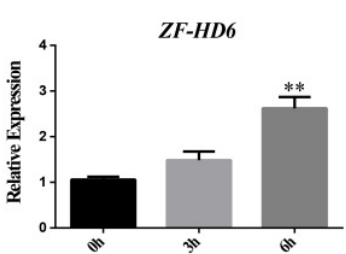

ZF-HD21

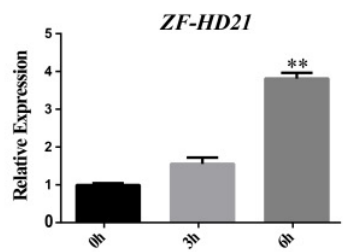

ZF-HD27

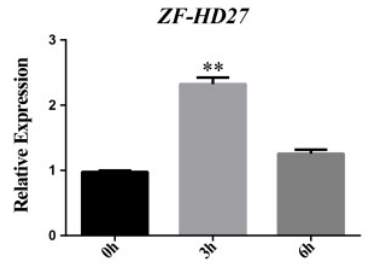

ZF-HD6
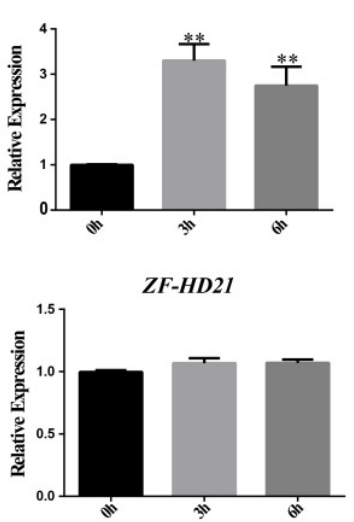

ZF-HD27

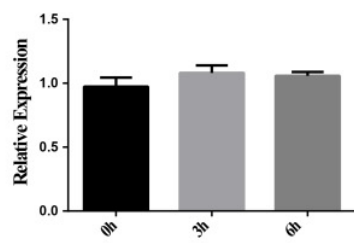

ZF-HD12

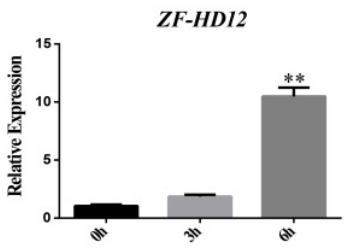

ZF-HD22

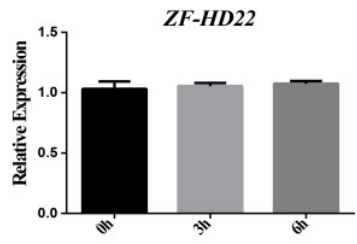

ZF-HD30

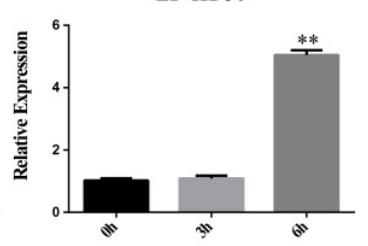

ZF-HD12
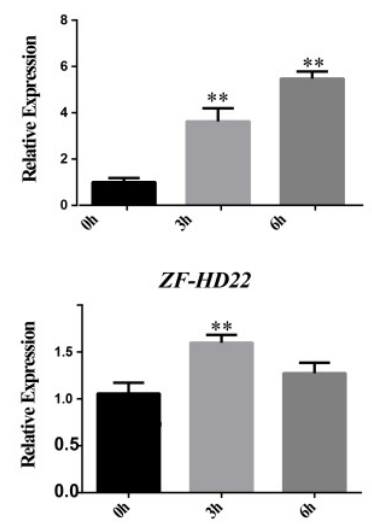

ZF-HD30

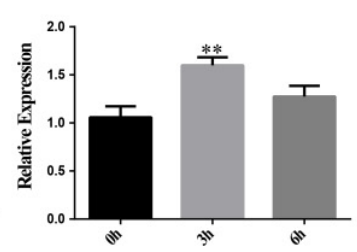

Figure 4. The expression patterns of selected NtZF-HD genes under (A) drought and (B) NaCl. The asterisks indicate significant statistical differences from the $0 \mathrm{~h}$ using the Student's $t$ test $(p<0.005)$. 


\subsection{Subcellular Localization of NtZF-HD Proteins}

According to previous reports, the ZF-HD protein, as a transcriptional activator, was located in the nucleus [18]. The prediction of subcellular localization indicated that almost all NtZF-HD proteins are located in the nucleus except for NtZF-HD14, which is located in the chloroplast and nucleus (Table S3).

To further investigate the subcellular localization of NtZF-HD proteins, NtZF-HD21 was selected to examine the subcellular localization. We constructed an NtZF-HD21-GFP fusion protein to detect the transient expression of NtZF-HD21 in the leaf of Nicotiana benthamiana. The NtZF-HD21-GFP fusion protein was predominantly observed in the nucleus and was able to overlap the staining signal of the dye 4,6-diamidino-2-phenylindole (DAPI) staining signal (Figure 5A). This result indicated that the NtZF-HD21 protein is a nuclear localization protein, which is consistent with its essential characteristics as a transcription factor.

A

A Merge
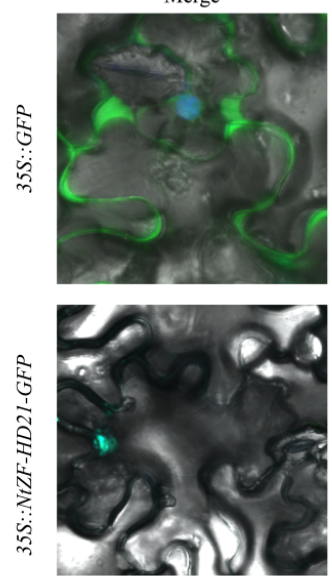

B

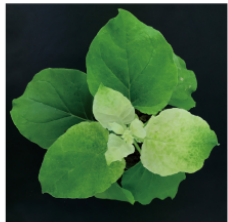

TRV2-PDS

D

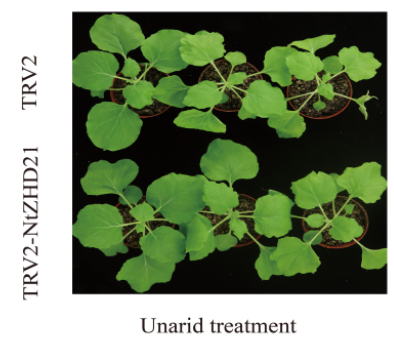

DAPI
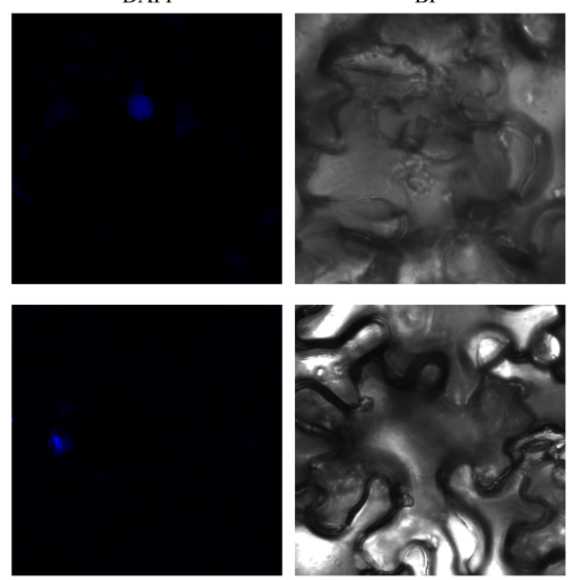

$\mathrm{BF}$

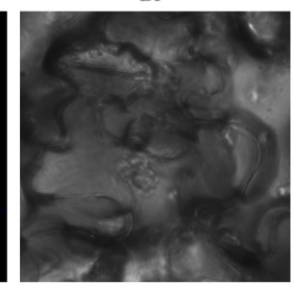

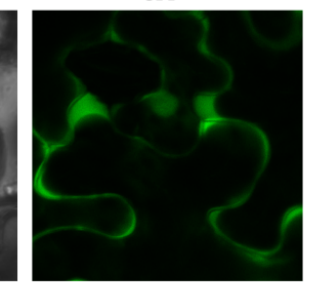

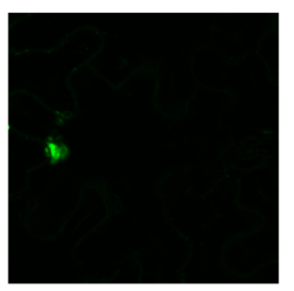

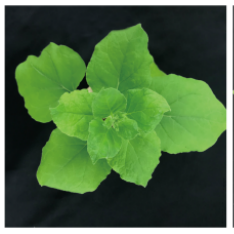

TRV2

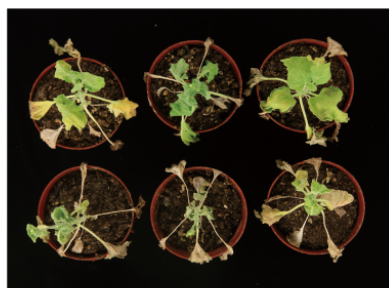

Drought treatment 3 weeks

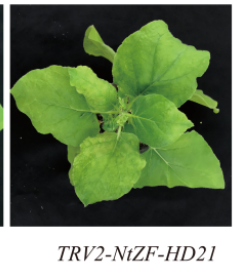

$\mathrm{C}$
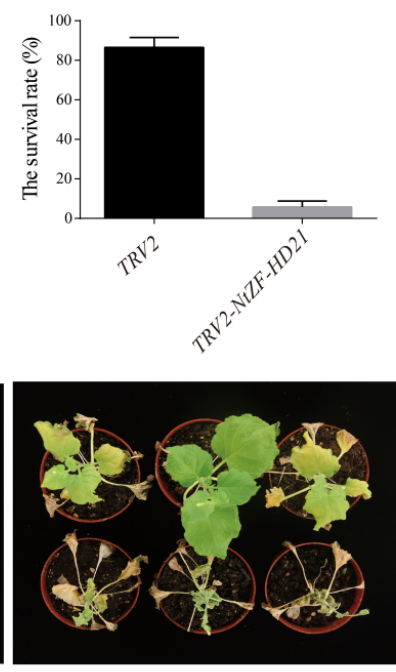

Rewatering treatment 3 days

Figure 5. The subcellular localization of NtZF-HD21 and virus-induced gene silencing (VIGS) of the NtZF-HD21 gene in Nicotiana benthamiana. (A) The subcellular localization of NtZF-HD21. The NtZF-HD21-GFP fusion construct and GFP gene driven by the CaMV-35S promoter were transiently expressed in Nicotiana benthamiana separately. 4,6-diamidino-2-phenylindole (DAPI) staining indicates the nucleus. (B) The phenotype of VIGS of the NtZF-HD21 gene in Nicotiana benthamiana. (C) The survival rate of silent plants and negative controlled plants. (D) The silence of NtZF-HD21 gene conferred the drought tolerance of tobacco. 


\subsection{Effect of NtZF-HD21 Gene Silencing on Drought Resistance}

Virus-induced gene silencing (VIGS) is a natural mechanism of plants' defense to virus invasion at post-transcriptional gene silencing [48]. VIGS is widely used in the study of gene function related to physiological pathways such as plant stress resistance, growth and development, and metabolic regulation [49]. To further investigate the functions of NtZF-HD numbers, NtZF-HD21 was selected to verify the function in response to drought stress by the VIGS system. The albino phenomenon of plants infected with TRV2-PDS as a positive control indicated that the recombinant plasmid was expressed in the plant (Figure 5B). There was no obvious difference in the phenotype between plants infected with TRV2-NtZF-HD21 silent plants and TRV2 negative-controlled plants. Subsequently, the silent plants and negative controlled plants were treated under drought stress for three weeks before re-watering treatment for three days. The results showed that the survival rate of silent plants was significantly lower compared with that of negative-controlled plants (Figure 5C,D), suggesting that the NtZF-HD21 gene was able to enhance the drought tolerance of tobacco.

\section{Discussion}

Previous research showed that ZF-HD transcription factors are only found in plants and play significant roles in plant developmental processes and various environmental stress responses [50]. A total of 32 members of the NtZF-HD genes were identified in the tobacco genome using BLASTP searches. The NtZF-HD genes were studied using phylogeny, gene structure, motif organization, synteny, cis-elements, and expression profiles.

The 32 NtZF-HD members were clustered into eight groups according to the phylogeny analysis of Arabidopsis and tomato NtZF-HD members. NtZF-HD genes of the same group contain similar gene structures and motif organization, suggesting that the previous evolutionary relationship and classification analysis of NtZF-HD genes were reliable. In addition, some NtZF-HD genes of the same group contained similar cis-elements types, implying that they may reflect similar functions in plant development and abiotic stress responses.

Increasing evidence indicates that $Z F-H D$ genes regulate the development of plants. The AtZF-HD5 in group III was reported to specifically regulate the size of leaves. NtZFHD22 and NtZF-HD26 were clustered with AtZF-HD5 and highly expressed in the leaves (Figures 1 and 4), suggesting its possible contribution to the growth and development of leaves. In addition, the AtZF-HD8 in group IV was reported to be involved in flower and leaf development. The NtZF-HD10 formed a collinear pair with AtZF-HD8, which expressed highly in flower and leaves, suggesting that they might have similar biological functions in flower and leaf development. Whereas, in the same group, AtZF-HD10 was reported to regulate the growth of root [19] and clustered with NtZF-HD4, which highly expressed in the roots, implying that NtZF-HD4 may be involved in root development.

Previous studies have indicated that a number of $Z F-H D$ genes mediated responses to drought and salinity stresses. AtZF-HD4 in group II, which has been reported to be involved in regulating drought and salt stress responses [25], was clustered together with NtZF-HD6 and NtZF-HD12. Interestingly, the promoter regions of NtZF-HD6 and NtZF-HD12 were found to harbor the cis-elements of the MYB binding site and TC-rich repeats element, and these genes could be induced under drought and salt treatments. These findings suggested that these genes may confer stress responses in tobacco. Notably, the overexpression of AtZF-HD1 altered ABA sensitivity, dehydration tolerance, and the expression levels of $\mathrm{ABA}$ /stress-regulated genes [22]. In group I, NtZF-HD5, NtZF-HD19, and NtZF-HD21 were clustered with AtZF-HD1. In the present study, these genes were found to harbor the ABRE element (Figure 1 and Table S6), suggesting that they might participate in drought response and ABA signaling. Moreover, NtZF-HD21 was up-regulated significantly under drought stress treatment. Furthermore, the VIGS experiment demonstrated that NtZF-HD21 can regulate the drought tolerance of tobacco plants (Figure 5). 
Overall, most NtZF-HD genes were expressed in different tissues and induced by drought and salinity abiotic stresses treatment in tobacco. Hence, we further suggested that NtZF-HD genes may play significant roles in various developmental processes and environmental stress responses in tobacco.

\section{Conclusions}

In this study, 32 ZF-HD genes were identified in Nicotiana tabacum. These genes were grouped into six groups based on the evolutionary analysis. Genes divided into the same group tend to have a similar exon-intron organization, motifs, and cis-acting elements. We also conducted expression patterns analysis of NtZF-HD genes, suggesting that they may be important for regulating plant responses to abiotic stresses and development. Notably, NtZF-HD21 was significantly induced by drought treatments, and it was able to enhance the drought tolerance of tobacco. Overall, the results from this study will be helpful to the further investigations of the function of tobacco NtZF-HD genes.

Supplementary Materials: The following are available online at https:/ /www.mdpi.com/2073-439 5/11/3/406/s1, Figure S1. Gene structure and motif organizations of NtZF-HD members in tobacco. Figure S2. The expression pattern of the selected NtZF-HD genes in different tissues. Table S1. The ZF-HD protein sequences of Arabidopsis, tomato, and tobacco, Table S2. The RT-qPCR primers of NtZF-HD genes, Table S3. The detailed information about NtZF-HD genes and NtZF-HD proteins in tobacco, Table S4. The amino acid sequences of motifs, Table S5. The syntenic analysis of NtZF-HD genes, Table S6. The predicted cis-acting elements in the promoter region of NtZF-HD genes.

Author Contributions: M.X., X.L. and Y.S. conceived the study and J.S. wrote the manuscript. J.S., Z.L. and Q.W. created the figures. A.D. and W.W. edited the manuscript. All authors have read and agreed to the published version of the manuscript.

Funding: This work was supported by the Mining and Application of Tobacco Gene Based on EMS Mutant Database (16102320200002) and the Science Foundation for Young Scholars of the Tobacco Research Institute of the Chinese Academy of Agricultural Sciences (2018B02).

Institutional Review Board Statement: Not applicable.

Informed Consent Statement: Not applicable.

Data Availability Statement: Data is contained within the article or supplementary material.

Conflicts of Interest: The authors declare that they have no competing interests.

\section{References}

1. Glazebrook, J. Genes controlling expression of defense responses in Arabidopsis-2001 status. Curr. Opin. Plant Biol. 2001, 4, 301-308. [CrossRef]

2. Singh, K.; Foley, R.C.; Onate-Sanchez, L. Transcription factors in plant defense and stress responses. Curr. Opin. Plant Biol. 2002, 5, 430-436. [CrossRef]

3. Wang, W.; Wu, P.; Li, Y.; Hou, X. Genome-wide analysis and expression patterns of ZF-HD transcription factors under different developmental tissues and abiotic stresses in Chinese cabbage. Mol. Genet. Genom. 2016, 291, 1451-1464. [CrossRef]

4. Krishna, S.S.; Majumdar, I.; Grishin, N.V. Structural classification of Zinc fingers: Survey and summary. Nucleic Acids Res. 2003, 31, 532-550. [CrossRef]

5. Ciftci-Yilmaz, S.; Mittler, R. The Zinc finger network of plants. Cell. Mol. Life Sci. 2008, 65, 1150-1160. [CrossRef]

6. Xie, M.M.; Sun, J.H.; Gong, D.P.; Kong, Y.Z. The roles of Arabidopsis C1-2i subclass of C2H2-type Zinc-finger transcription factors. Genes 2019, 10, 653. [CrossRef]

7. Hu, W.; dePamphilis, C.W.; Ma, H. Phylogenetic analysis of the plant-specific Zinc finger-homeobox and mini Zinc finger gene families. J. Integr. Plant Biol. 2008, 50, 1031-1045. [CrossRef] [PubMed]

8. Mukherjee, K.; Brocchieri, L.; Burglin, T.R. A comprehensive classification and evolutionary analysis of plant homeobox genes. Mol. Biol. Evol. 2009, 26, 2775-2794. [CrossRef]

9. Gehring, W.J.; Affolter, M.; Burglin, T. Homeodomain proteins. Annu. Rev. Biochem. 1994, 63, 487-526. [CrossRef] [PubMed]

10. Wolberger, C. Homeodomain interactions. Curr. Opin. Struct. Biol. 1996, 6, 62-68. [CrossRef]

11. Ariel, F.D.; Manavella, P.A.; Dezar, C.A.; Chan, R.L. The true story of the HD-ZIP family. Trends Plant Sci. 2007, 12, 419-426. [CrossRef] 
12. Kawagashira, N.; Ohtomo, Y.; Murakami, K.; Matsubara, K.; Kawai, J.; Carninci, P.; Hayashizaki, Y.; Kikuchi, S.; Higo, K. Multiple Zinc finger motifs with comparison of plant and insect. Genom. Inform. 2001, 12, 368-369.

13. Englbrecht, C.C.; Schoof, H.; Bohm, S. Conservation, diversification and expansion of $\mathrm{C}_{2} \mathrm{H}_{2}$ zinc finger proteins in the Arabidopsis thaliana genome. BMC Genom. 2004, 5, 39. [CrossRef]

14. Windhovel, A.; Hein, I.; Dabrowa, R.; Stockhaus, J. Characterization of a novel class of plant homeodomain proteins that bind to the C4 phosphoenolpyruvate carboxylase gene of Flaveria trinervia. Plant Mol. Biol. 2001, 45, 201-214. [CrossRef]

15. Galvao, V.C.; Horrer, D.; Kuttner, F.; Schmid, M. Spatial control of flowering by della proteins in Arabidopsis thaliana. Development 2012, 139, 4072-4082. [CrossRef]

16. Porri, A.; Torti, S.; Romera-Branchat, M.; Coupland, G. Spatially distinct regulatory roles for gibberellins in the promotion of flowering of Arabidopsis under long photoperiods. Development 2012, 139, 2198-2209. [CrossRef] [PubMed]

17. Shalmani, A.; Muhammad, I.; Sharif, R.; Zhao, C.; Ullah, U.; Zhang, D.; Jing, X.Q.; Amin, B.; Jia, P.; Tahir, M.M.; et al. Zinc finger-homeodomain genes: Evolution, functional differentiation, and expression profiling under flowering-related treatments and abiotic stresses in plants. Evol. Bioinform. 2019, 15, 1-16. [CrossRef]

18. Hong, S.Y.; Kim, O.K.; Kim, S.G.; Yang, M.S.; Park, C.M. Nuclear import and DNA binding of the ZHD5 transcription factor is modulated by a competitive peptide inhibitor in Arabidopsis. J. Biol. Chem. 2011, 286, 1659-1668. [CrossRef] [PubMed]

19. Perrella, G.; Davidson, M.L.H.; O’Donnell, L.; Nastase, A.M.; Herzyk, P.; Breton, G.; Pruneda-Paz, J.L.; Kay, S.A.; Chory, J.; Kaiserli, E. Zinc-finger interactions mediate transcriptional regulation of hypocotyl growth in Arabidopsis. Proc. Natl. Acad. Sci. USA 2018, 115, E4503-E4511. [CrossRef]

20. Khatun, K.; Nath, U.K.; Robin, A.H.K.; Park, J.I.; Lee, D.J.; Kim, M.B.; Kim, C.K.; Lim, K.B.; Nou, I.S.; Chung, M.Y. Genome-wide analysis and expression profiling of Zinc finger homeodomain (ZHD) family genes reveal likely roles in organ development and stress responses in tomato. BMC Genom. 2017, 18, 695. [CrossRef] [PubMed]

21. Liu, M.; Wang, X.; Sun, W.; Ma, Z.; Zheng, T.; Huang, L.; Wu, Q.; Tang, Z.; Bu, T.; Li, C.; et al. Genome-wide investigation of the ZF-HD gene family in tartary buckwheat (Fagopyrum tataricum). BMC Plant Biol. 2019, 19, 248. [CrossRef]

22. Tran, L.S.P.; Nakashima, K.; Sakuma, Y.; Osakabe, Y.; Qin, F.; Simpson, S.D.; Maruyama, K.; Fujita, Y.; Shinozaki, K.; YamaguchiShinozaki, K. Co-expression of the stress-inducible Zinc finger homeodomain ZFHD1 and NAC transcription factors enhances expression of the ERD1 gene in Arabidopsis. Plant. J. 2007, 49, 46-63. [CrossRef] [PubMed]

23. Wang, H.; Yin, X.; Li, X.; Wang, L.; Zheng, Y.; Xu, X.; Zhang, Y.; Wang, X. Genome-wide identification, evolution and expression analysis of the grape (Vitis vinifera L.) Zinc finger-homeodomain gene family. Int. J. Mol. Sci. 2014, 15, 5730-5748. [CrossRef] [PubMed]

24. Bhattacharjee, A.; Jain, M. Homeobox genes as potential candidates for crop improvement under abiotic stress. Plant. Acclim. Environ. Stress 2013, 163-176. [CrossRef]

25. Barth, O.; Vogt, S.; Uhlemann, R.; Zschiesche, W.; Humbeck, K. Stress induced and nuclear localized hipp26 from Arabidopsis thaliana interacts via its heavy metal associated domain with the drought stress related Zinc finger transcription factor ATHB29. Plant Mol. Biol. 2009, 69, 213-226. [CrossRef] [PubMed]

26. Park, H.C.; Kim, M.L.; Lee, S.M.; Bahk, J.D.; Yun, D.J.; Lim, C.O.; Hong, J.C.; Lee, S.Y.; Cho, M.J.; Chung, W.S. Pathogen-induced binding of the soybean zinc finger homeodomain proteins GmZF-HD1 and GmZF-HD2 to two repeats of atta homeodomain binding site in the Calmodulin Isoform 4 (GmCaM4) promoter. Nucleic Acids Res. 2007, 35, 3612-3623. [CrossRef] [PubMed]

27. Jain, M.; Tyagi, A.K.; Khurana, J.P. Genome-wide identification, classification, evolutionary expansion and expression analyses of homeobox genes in rice. FEBS J. 2008, 275, 2845-2861. [CrossRef]

28. Ma, J.J.; Zheng, L.W.; Zhao, C.D.; Li, G.F.; Shen, Y.W.; An, N.; Zhang, D.; Han, M.Y. Genome-wide identification and expression analysis of half-size ABCG genes in Malus $\times$ domestica. Hortic Plant. J. 2018, 4, 45-54. [CrossRef]

29. Sierro, N.; Battey, J.N.; Ouadi, S.; Bakaher, N.; Bovet, L.; Willig, A.; Goepfert, S.; Peitsch, M.C.; Ivanov, N.V. The tobacco genome sequence and its comparison with those of tomato and potato. Nat. Commun. 2014, 5, 3833. [CrossRef] [PubMed]

30. Edwards, K.D.; Fernandez-Pozo, N.; Drake-Stowe, K.; Humphry, M.; Evans, A.D.; Bombarely, A.; Allen, F.; Hurst, R.; White, B.; Kernodle, S.P.; et al. A reference genome for Nicotiana tabacum enables map-based cloning of homeologous loci implicated in nitrogen utilization efficiency. BMC Genom. 2017, 18, 448. [CrossRef] [PubMed]

31. Fernandez-Pozo, N.; Menda, N.; Edwards, J.D.; Saha, S.; Tecle, I.Y.; Strickler, S.R.; Bombarely, A.; Fisher-York, T.; Pujar, A.; Foerster, H.; et al. The Sol Genomics Network (SGN)-from genotype to phenotype to breeding. Nucleic Acids Res. 2015, 43, D1036-D1041. [CrossRef]

32. Poole, R.L. The TAIR database. Methods Mol. Biol. 2007, 406, 179-212. [PubMed]

33. Finn, R.D.; Coggill, P.; Eberhardt, R.Y.; Eddy, S.R.; Mistry, J.; Mitchell, A.L.; Potter, S.C.; Punta, M.; Qureshi, M.; Sangrador-Vegas, A.; et al. The Pfam protein families database: Towards a more sustainable future. Nucleic Acids Res. 2016, 44, D279-D285. [CrossRef] [PubMed]

34. Letunic, I.; Doerks, T.; Bork, P. SMART: Recent updates, new developments and status in 2015. Nucleic Acids Res. 2015, 43, D257-D260. [CrossRef]

35. Garg, V.K.; Avashthi, H.; Tiwari, A. MFPPI-Multi FASTA protparam interface. Bioinformation 2016, 12, 74-77. [CrossRef]

36. Kumar, S.; Stecher, G.; Li, M.; Knyaz, C.; Tamura, K. MEGA X: Molecular evolutionary genetics analysis across computing platforms. Mol. Biol. Evol. 2018, 35, 1547-1549. [CrossRef] [PubMed] 
37. Hu, B.; Jin, J.P.; Guo, A.Y.; Zhang, H.; Luo, J.C.; Gao, G. GSDS 2.0: An upgraded gene feature visualization server. Bioinformatics 2015, 31, 1296-1297. [CrossRef] [PubMed]

38. Bailey, T.L.; Johnson, J.; Grant, C.E.; Noble, W.S. The MEME suite. Nucleic Acids Res. 2015, 43, W39-W49. [CrossRef]

39. Lescot, M.; Dehais, P.; Thijs, G.; Marchal, K.; Moreau, Y.; Van de Peer, Y.; Rouze, P.; Rombauts, S. PlantCARE, a database of plant cis-acting regulatory elements and a portal to tools for in silico analysis of promoter sequences. Nucleic Acids Res. 2002, 30, 325-327. [CrossRef]

40. Chen, C.J.; Chen, H.; Zhang, Y.; Thomas, H.R.; Frank, M.H.; He, Y.H.; Xia, R. TBtools: An integrative toolkit developed for interactive analyses of big biological data. Mol. Plant 2020, 13, 1194-1202. [CrossRef]

41. Khatun, K.; Robin, A.H.K.; Park, J.I.; Ahmed, N.U.; Kim, C.K.; Lim, K.B.; Kim, M.B.; Lee, D.J.; Nou, I.S.; Chung, M.Y. Genome-wide identification, characterization and expression profiling of LIM family genes in Solanum lycopersicum L. Plant Physiol Biochem. 2016, 108, 177-190. [CrossRef] [PubMed]

42. Ren, A.; Ahmed, R.I.; Chen, H.; Han, L.; Sun, J.; Ding, A.; Guo, Y.; Kong, Y. Genome-wide identification, characterization and expression patterns of the pectin methylesterase inhibitor genes in sorghum bicolor. Genes 2019, 10, 755. [CrossRef] [PubMed]

43. Untergasser, A.; Cutcutache, I.; Koressaar, T.; Ye, J.; Faircloth, B.C.; Remm, M.; Rozen, S.G. Primer3-new capabilities and interfaces. Nucleic Acids Res. 2012, 40, e115. [CrossRef] [PubMed]

44. Livak, K.J.; Schmittgen, T.D. Analysis of relative gene expression data using real-time quantitative PCR and the 2 (-Delta Delta C (T)) method. Methods 2001, 25, 402-408. [CrossRef] [PubMed]

45. Chou, K.C.; Shen, H.B. Plant-mPLoc: A top-down strategy to augment the power for predicting plant protein subcellular localization. PLoS ONE 2010, 5, e11335. [CrossRef] [PubMed]

46. Li, X.; Guo, C.; Ahmad, S.; Wang, Q.; Yu, J.; Liu, C.; Guo, Y. Systematic analysis of MYB family genes in potato and their multiple roles in development and stress responses. Biomolecules 2019, 9, 317. [CrossRef]

47. Fernandez-Pozo, N.; Rosli, H.G.; Martin, G.B.; Mueller, L.A. The SGN VIGS tool: User-friendly software to design virus-induced gene silencing (VIGS) constructs for functional genomics. Mol. Plant 2015, 8, 486-488. [CrossRef] [PubMed]

48. Gao, X.; Shan, L. Functional Genomic Analysis of Cotton Genes with Agrobacterium-Mediated Virus-Induced Gene Silencing. Methods Mol. Biol. 2013, 975, 157-165. [CrossRef] [PubMed]

49. Burch-Smith, T.M.; Anderson, J.C.; Martin, G.B.; Dinesh-Kumar, S.P. Applications and advantages of virus-induced gene silencing for gene function studies in plants. Plant J. 2010, 39, 734-746. [CrossRef] [PubMed]

50. Li, Y.; Bai, B.; Wen, F.; Zhao, M.; Xia, Q.; Yang, D.H.; Wang, G. Genome-wide identification and expression analysis of HDZIP I gene subfamily in Nicotiana tabacum. Genes 2019, 10, 575. [CrossRef] 